\title{
Determinación de nitrito como metabolito estable del óxido nítrico en el líquido sinovial de articulación metacarpofalángica equina ${ }^{\#}$
}

\author{
Nitrite determination as a stable metabolite of nitric oxide in synovial fluid of equine \\ metacarpophalangeal joint
}

\author{
Adarmes H, JP Solís, A Müller, M Galleguillos* \\ Facultad de Ciencias Veterinarias y Pecuarias, Universidad de Chile, Santiago, Chile.
}

\begin{abstract}
SUMMARY
The striking feature of osteoarthritis (OA) is fibrilation and loss of articular cartilage. Nitric oxide (NO*) has been involved as an important biological mediator of $\mathrm{OA}$ and its production is significantly higher in $\mathrm{OA}$ cartilage. $\mathrm{NO}^{*}$ suppresses cartilagenous matrix synthesis and induces condrocytes apoptosis in articular cartilage. The NO production was indirectly determined by measuring nitrite by Griess reaction. Nitrite concentration of synovial fluid was compared in different equine metacarpophalangic joint conditions. Joint classification and age determination were carried out by macroscopic examination. It was considered a normal joint group $\mathrm{NN}(\mathrm{n}=47)$ the one without any visual damage of articular structures. The damaged groups considered a) a group with a congestive synovial membrane without cartilage damage NA $(n=25)$, b) a group with damaged cartilage and normal synovial membrane AN ( $\mathrm{n}=23)$ and c) a group with damaged cartilage and congestive synovial membrane AA $(n=15)$. A damaged cartilage showed erosions and multiple wear lines and a damaged synovial membrane showed a congestive appearance. There were no significant differences in nitrite concentration between the analyzed groups. Only in the NA group a non significant increase $(44.4 \pm 32.6 \mu \mathrm{mol} / \mathrm{L})$ was detected, that was possibly associated to the absence of cartilage loss and so with a major response to proinflammatory stimulation originated in the congestive synovial membrane. Nitrite concentration decreased in a non significant way in the damaged cartilage and synovial membrane group AA $(26.3 \pm 22.1 \mu \mathrm{mol} / \mathrm{L})$ which might be explained by cartilage loss in this in vivo model.
\end{abstract}

Palabras clave: líquido sinovial, nitrito, equino.

Key words: synovial fluid, nitrite, equine.

\section{INTRODUCCIÓN}

El óxido nítrico (NO*) es un radical libre que participa en la patogenia de muchas enfermedades que incluyen la osteoartritis en especies como el hombre, el perro y el equino, debido a que es sintetizado por condrocitos y sinoviocitos como consecuencia del estímulo de citoquinas proinflamatorias (Moshage 1997, von Rechenberg y col 2000, Davis y col 2001, Spreng y col 2001, Tomita y col 2001, Tung y col 2002). El NO• se sintetiza a partir de la L-arginina, por la actividad de la enzima óxido nítrico sintasa (NOS), que posee tres isoformas, NOS-I, NOS-II y NOS-III (Murrell y col 1995, Moshage 1997, Ralston 1997, Clancy y col 1998, Stichtenoth y Frölich 1998, Miranda y col 2001, Ersoy y col 2002, Lee y col 2002). La NOS-I y la NOS-III son descritas como isoformas constitutivas (cNOS) y pueden ser objeto de regulación génica (Förstermann y col 1998), mientras que la NOS-II representa la isoforma inducible (iNOS) ya que se expresa en células estimuladas por diferentes citoquinas proinflamatorias como la interleuquina-1 (IL-1), interferon gamma (INF$\gamma)$ y el factor de necrosis tumoral- $\alpha$ (TNF- $\alpha)$. La NOS-II

Aceptado: 20.05.2009.

\# Proyecto DID I-14-2/2001. Universidad de Chile.

* Casilla 2 correo 15, La Granja, Santiago, Chile; mgallegu@uchile.cl produce concentraciones mucho más elevadas de $\mathrm{NO}^{*}$ con respecto a la actividad de NOS-I y NOS-III (Murrell y col 1995, Moshage 1997, Ralston 1997, Stichtenoth y Frölich 1998, Davis y col 2001, Tomita y col 2001).

Los condrocitos constituirían la fuente más importante de NO^ en la articulación (Jang y Murrell 1998). También se ha establecido que el cartílago osteoartrítico equino produce mayores concentraciones de $\mathrm{NO}^{*}$ (van der Harst y col 2006) y que los condrocitos superficiales de cartílago normal humano sintetizan 2 a 3 veces más NO* que los de zonas profundas al ser estimulados con IL-1. También se ha demostrado que esta respuesta disminuye a través de la edad (Häuselmann y col 1998). La osteoartritis (OA) o Enfermedad Degenerativa Articular (EDA) se caracteriza por la pérdida focal de cartílago debido al estímulo de las vías catabólicas inducido por la IL-1 y el TNF- $\alpha$ (Mazzetti y col 2001). Se produce pérdida de la función del cartílago, originada por su erosión y por la reducción del contenido de proteoglicanos y de colágeno, acompañado de cambios tanto en la estructura como en la agregación de estas moléculas (McIlwraith 1996).

En relación al papel que desempeña el $\mathrm{NO}^{\bullet}$ en el cartílago existen planteamientos contradictorios, ya que por un lado participaría como modulador endógeno del recambio de la matriz extracelular en diferentes zonas del cartílago articular (Häuselmann y col 1998) e incluso 
podría proteger de la degradación del agrecán (Bird y col 2000). Por otro lado, también se ha sugerido que el NO• sería uno de los responsables de la destrucción del cartílago en la osteoartritis de rodilla humana (Karan y col 2003) y podría existir una relación entre la apoptosis de condrocitos en el cartílago equino y el incremento de la concentración de NO• (van't Hof y col 2000, Kim y col 2003).

La cuantificación de NO• es difícil en medios biológicos, debido a su corta vida media (de 6 a 10 segundos) y a sus bajas concentraciones y por eso se cuantifican sus metabolitos estables: nitrito y nitrato (Ralston 1997, Stichtenoth y Frölich 1998, Miranda y col 2001, Spreng y col 2001, Ersoy y col 2002). El nitrito es el único producto estable resultante de la autooxidación del $\mathrm{NO}^{\bullet}$ en soluciones acuosas como líquido cefalorraquídeo y líquido sinovial. El nitrato, en cambio, se forma por la reacción entre el $\mathrm{NO}^{\circ} \mathrm{y}$ la oxihemoglobina, lo que implica que el nitrato existe en mayor proporción en plasma y suero (Stichtenoth y Frölich 1998, Miranda y col 2001, Ersoy y col 2002).

Si bien los modelos in vitro señalan el incremento de la síntesis de $\mathrm{NO}^{*}$ en el cartílago osteoartrítico como consecuencia del estímulo con IL-1, posiblemente en un modelo articular in vivo se podría esperar que la pérdida de cartílago y la apoptosis de condrocitos (van't Hof y col 2000, Kim y col 2003) provocarían una menor síntesis de $\mathrm{NO}^{\bullet} \mathrm{y}$, por ende, una menor concentración de nitrito en el líquido sinovial. En este trabajo se utilizó el líquido sinovial de la articulación metacarpofalángica equina obtenido inmediatamente después del sacrificio de los animales. En estas condiciones, la concentración de nitrito del líquido sinovial reflejaría la síntesis de $\mathrm{NO}^{*}$ por el conjunto de estructuras articulares y particularmente del cartílago articular. Se buscó establecer si la concentración de nitrito del líquido sinovial se relaciona con diferentes estados de alteración articular, tanto de la membrana sinovial como del cartílago articular, y así establecer si la concentración de nitrito podría constituir un marcador de diferentes estados de alteración articular.

\section{MATERIAL Y MÉTODOS}

\section{MATERIAL BIOLÓGICO}

Se obtuvo líquido sinovial (LS) por artrocentesis aséptica, desde la articulación metacarpofalángica de equinos criollos de matadero, inmediatamente después del beneficio de los animales, a los que se determinó su edad por cronometría dentaria. La punción se realizó por la cara palmar (recesos sinoviales) utilizando jeringas de $5 \mathrm{~mL}$ con agujas $21 \mathrm{G}$ x 11/2. Se seleccionaron articulaciones metacarpofalángicas normales y otras con alteración del cartílago y/o de la membrana sinovial, en base a la inspección visual post mortem. Cada articulación metacarpofalángica seleccionada para este estudio proviene de equinos diferentes. Se consideró como normal (NN) a aquellas articulaciones sin alteraciones visibles de esos tejidos articulares $(n=47)$, es decir, con un cartílago de superficie lisa y brillante, de color blanco nacarado y una membrana sinovial sin signos de congestión. Las articulaciones alteradas fueron divididas en a) aquellas que presentaron sólo congestión de la membrana sinovial (NA) $(n=25), b)$ aquellas que presentaron sólo daño del cartílago articular como focos de erosión y líneas de roce (AN) $(n=23)$ y c) aquellas que presentaron tanto daño del cartílago (focos de erosión y líneas de roce) como congestión de la membrana sinovial (AA) $(n=15)$. Para la selección de las articulaciones metacarpofalángicas, tanto normales como alteradas, se consideró que el LS debía presentar un color amarillo claro, translúcido y sin derrame sanguinolento, de lo contrario la articulación con su líquido sinovial fueron eliminados.

Las muestras de LS obtenidas se centrifugaron a $1.000 \times$ g durante 20 minutos. Los sobrenadantes se alicuotaron y se conservaron a $-20^{\circ} \mathrm{C}$ hasta su procesamiento.

\section{DETERMINACIÓN DE NITRITO}

En el LS se determinó la concentración de nitrito utilizando la reacción de Griess (Miranda y col 2001), que detecta específicamente nitrito y no nitrato (Moshage 1997, Miranda y col 2001). El reactivo de Griess se prepara mezclando una solución de N-1-(naftil) etilendiamina dihidrocloruro $(0,1 \% \mathrm{p} / \mathrm{v}$ en $5 \%$ de $\mathrm{HCl})$ con una solución de sulfanilamida $(2 \% \mathrm{p} / \mathrm{v}$ en $5 \% \mathrm{HCl})$. Ambas soluciones son estables por varios meses a $4{ }^{\circ} \mathrm{C}$ protegidas de la luz; éstas se mezclaron inmediatamente antes de realizar la reacción colorimétrica.

Debido a que esta reacción se podría alterar por compuestos como los S-nitrosotioles y derivados de la L-arginina, las muestras de LS se desproteinizaron (Miranda y col 2001) por dilución 1:7 con etanol. Las proteínas precipitadas se separaron por centrifugación a $1.000 \times \mathrm{g}$ durante 20 minutos. La reacción colorimétrica se obtiene agregando a $300 \mu \mathrm{L}$ del sobrenadante desproteinizado, $300 \mu \mathrm{L}$ del reactivo de Griess y se completa un volumen final de 900 $\mu \mathrm{L}$ con agua destilada, estableciéndose su absorbancia a $540 \mathrm{~nm}$. Como estándar se utilizó $\mathrm{NaNO}_{2}$ 0,1 mM en un rango entre $0,9 \mu$ moles y $10 \mu$ moles. La concentración se expresó como $\mu$ moles de nitrito/L.

La reacción de Griess se basa en la formación de un cromóforo por la reacción de sulfanilamida con nitrito en medio ácido, seguido de un acoplamiento con aminas bicíclicas tales como el N-1-(naftil) etilendiamina dihidrocloruro.

\section{DETERMINACIÓN DE PROTEÍNAS}

Los objetivos de esta determinación fueron a) establecer la ausencia de proteínas en el sobrenadante, luego de desproteinizar el líquido sinovial con etanol para la determinación de nitrito, b) eliminar aquellos líquidos sinoviales cuya concentración de proteínas estuviese muy 
por encima de los $20 \mathrm{~g} / \mathrm{L}$ que indicaría una inflamación articular intensa (Adarmes y col 2008) y c) establecer alguna relación entre la concentración de proteínas con la de nitritos en el líquido sinovial.

Para esta determinación, el LS o el sobrenadante obtenido luego de la desproteinización fueron diluidos en una relación 1:10 con agua destilada y la concentración de proteínas se determinó por el método espectrofotométrico de Lowry y col (1951) a 750 nm, utilizando seroalbúmina de bovino (BSA) como estándar en un rango entre $5 \mu \mathrm{g}$ y $50 \mu \mathrm{g}$. Los resultados se expresan como g/L.

\section{ANÁLISIS ESTADÍSTICO}

A los valores promedio de nitrito y de proteína de las muestras se les estableció un nivel de significancia $(\mathrm{P}<0,05)$ a través de un análisis de varianza y posteriormente por la prueba de Tukey (Infostat 2004).

\section{RESULTADOS Y DISCUSIÓN}

En este trabajo se formaron cuatro grupos articulares a partir de diferencias macroscópicas evidenciables a simple vista, tanto a nivel del cartílago articular como de la membrana sinovial. Esta diferenciación entre los grupos se refuerza en el hecho de que las lesiones del cartílago articular aparecen en aquellos equinos de mayor edad (AN 9,9 \pm 4,2 años y AA 10,7 \pm 3,8 años).

Se evaluó la concentración de $\mathrm{NO}_{2}{ }^{-}$en el líquido sinovial equino inmediatamente después del beneficio de los animales. El $\mathrm{NO}^{\bullet}$ es extremadamente lábil ya que interacciona rápidamente con $\mathrm{O}_{2}$ para generar una variedad de óxidos de nitrógeno, que incluyen los nitritos y nitratos como los productos estables más importantes del $\mathrm{NO}^{\bullet}$ en los fluidos biológicos. $\mathrm{El} \mathrm{NO}_{2}^{-}$se midió a través de la reacción de Griess, que es el método más utilizado, debido a que el $\mathrm{NO}_{2}^{-}$es el único producto estable que se genera por la descomposición del $\mathrm{NO}^{\bullet}$ en soluciones acuosas in vitro. Sin embargo, también se ha detectado $\mathrm{NO}_{3}{ }^{-}$en forma predominante, en fluidos extracelulares como plasma $\mathrm{u}$ orina (Nagano 1999). En la selección de las muestras se descartaron aquellos líquidos sinoviales con derrame sanguinolento, debido a que la oxihemoglobina podría reaccionar con el $\mathrm{NO}_{2}{ }^{-}$para generar metahemoglobina y de ese modo afectar el contenido de $\mathrm{NO}_{2}^{-}$(Nagano 1999). Además, se descartaron aquellos líquidos sinoviales que presentaron turbidez.

En nuestros resultados no se encontró diferencia significativa para los valores de proteína y de nitrito en el líquido sinovial de los distintos grupos articulares seleccionados, ni tampoco alguna relación entre ambas concentraciones (cuadro 1).

Sólo se pudo apreciar un incremento no significativo de la concentración de nitrito en las articulaciones con daño en alguna de la estructuras articulares (NA y AN). La tendencia de los nitritos a aumentar en el grupo NA
Cuadro 1. Clasificación articular, edad promedio de los equinos (años), concentración de proteínas (g/L) y de nitrito $(\mu \mathrm{mol} / \mathrm{L})$ del líquido sinovial de la articulación metacarpofalángica equina.

Joint classification, average age (years), synovial fluid concentration of proteins $(\mathrm{g} / \mathrm{L})$ and nitrite $(\mu \mathrm{mol} / \mathrm{L})$ in equine metacarpophalangeal joint.

\begin{tabular}{cccc}
\hline Articulación & $\begin{array}{c}\text { Edad Promedio } \\
\pm \mathrm{DE} \\
(\mathrm{años})\end{array}$ & $\begin{array}{c}\text { Proteínas } \\
\pm \mathrm{DE} \\
(\mathrm{g} / \mathrm{L})\end{array}$ & $\begin{array}{c}\text { Nitrito } \\
\pm \mathrm{DE} \\
(\mu \mathrm{mol} / \mathrm{L})\end{array}$ \\
\hline $\mathrm{NN}$ & $5,3 \pm 3,6$ & $15,2 \pm 6,3$ & $29,4 \pm 27,9$ \\
$\mathrm{NA}$ & $4,4 \pm 3,1$ & $13,4 \pm 8,4$ & $44,4 \pm 32,6$ \\
AN & $9,9 \pm 4,2$ & $14,4 \pm 7,4$ & $32,7 \pm 27,0$ \\
AA & $10,7 \pm 3,8$ & $10,8 \pm 5,4$ & $26,3 \pm 26,1$ \\
\hline
\end{tabular}

NN: Cartílago y membrana sinovial macroscópicamente normales. NA: Cartílago normal y membrana sinovial congestiva. AN: Cartílago alterado y membrana sinovial normal. AA: Cartílago alterado y membrana sinovial congestiva.

(44,4 \pm 32,6 $\mu \mathrm{mol} / \mathrm{L})$ se podría asociar a que el estímulo proinflamatorio de la membrana sinovial congestiva encontraría una masa de cartílago normal, lográndose estimular una mayor síntesis de NO` por los condrocitos. Este modelo NA se podría asemejar con una sinovitis leve, dado que la concentración de proteínas en ninguno de los casos presenta diferencias significativas y no representa valores asociados a inflamaciones más intensas (Adarmes y col 2008).

En el grupo AN que representaría una osteoartritis moderada, básicamente por el daño en el cartílago articular, también aparece una tendencia a aumentar del contenido de nitrito $(32,7 \pm 27 \mu \mathrm{mol} / \mathrm{L})$ al igual que lo descrito en el trabajo de von Rechenberg y col (2000). Sin embargo, el incremento no tan elevado como en el grupo NA podría reflejar una menor masa de cartílago asociada a la pérdida de tejido, a pesar de que se ha descrito una mayor síntesis de $\mathrm{NO}^{\bullet}$ en el cartílago equino osteoartrítico in vitro (van der Harst y col 2006).

En el caso del grupo (AA) que representaría una osteoartritis severa, se encontró una disminución no significativa del contenido de nitrito $(26,3 \pm 26,1 \mu \mathrm{mol} / \mathrm{L})$ a pesar del estímulo que podría generar la membrana sinovial alterada a través de citoquinas proinflamatorias. Esta tendencia a disminuir de los nitritos en este grupo AA se podría relacionar con dos aspectos: a) la mayor pérdida de tejido articular reflejada por el mayor daño del tejido cartilaginoso y b) al hecho de que se ha observado una disminución de la síntesis de nitrito por el cartílago equino a través de la edad (van der Harst y col 2006). Este grupo AA constituye el grupo de mayor edad (10,7 $\pm 3,8$ años) y este factor podría ser relevante en este resultado. La diferencia no significativa en la concentración de nitrito del grupo AA con respecto al grupo AN no se debería a la edad, que es ligeramente inferior en este último grupo 
$(9,9 \pm 4,2$ años), por lo que la diferencia se podría asociar a una mayor cronicidad del cuadro patológico en el grupo AA con la consiguiente pérdida de cartílago. También se ha descrito que la disminución de nitrito en el líquido sinovial se podría explicar por el incremento de la apoptosis de condrocitos, que constituye una de las causas principales de los cambios patológicos dependientes de la edad (Hashimoto y col 1998).

Von Rechenberg y col (2000) en un modelo in vitro demostraron a través de la reacción de Griess que la concentración de $\mathrm{NO}^{*}$ fue mayor en el medio de cultivo de explantes de cartílago articular equino que en el de membrana sinovial, lo que indicaría una mayor actividad del condrocito en la producción de $\mathrm{NO}^{*}$. Además, en ese trabajo se compararon tejidos articulares normales con aquellos provenientes de osteartritis moderada y osteoartritis severa, detectándose en el caso de la osteoartritis moderada incremento significativo de $\mathrm{NO}^{*}$ en los explantes de cartílago y de membrana sinovial. Para realizar la comparación en dicho trabajo se utilizaron 25 $\mathrm{mg}$ de tejido hidratado/mL de medio de cultivo, tanto para cartílago como para membrana sinovial, de modo que la concentración de nitrito del medio de cultivo sólo refleja la actividad metabólica de esa cantidad de tejido. Por el contrario, nuestro trabajo refleja el contenido total de nitrito del líquido sinovial producido por el conjunto de estructuras articulares in vivo.

Se concluyó que en estados de alteración severa de la articulación (AA) disminuyó la concentración de NO", posiblemente debido a una menor capacidad de síntesis provocada por la pérdida de tejido cartilaginoso y/o por apoptosis de condrocitos, lo que coincide con los equinos de mayor edad. Además, en los estados intermedios de alteración articular (AN y NA) aumentó la concentración de $\mathrm{NO}^{*}$ en forma independiente de la edad. No se encontró relación entre las concentraciones de proteína y de nitrito en el líquido sinovial.

Para responder al efecto de la edad in vivo se podría evaluar la concentración de nitrito en el líquido sinovial de equinos de edades diferentes con sus articulaciones normales. Por otro lado, el inconveniente del modelo in vitro es que al comparar cantidades similares de condrocitos o de cartílago en un medio de cultivo no se refleja la actividad total del conjunto de estructuras articulares del modelo in vivo.

\section{RESUMEN}

La osteoartritis $(\mathrm{OA})$ se caracteriza por fibrilación y erosión del cartílago articular. El óxido nítrico (NO*) sería un importante mediador biológico de la OA y su producción es significativamente mayor en el cartílago OA. El NO• inhibe la síntesis de matriz cartilaginosa e induce la apoptosis en el cartílago articular. Se determinó indirectamente la producción de NO a través de la concentración de nitrito utilizando la reacción de Griess. Se comparó la concentración de nitrito del líquido sinovial de la articulación metacarpofalángica equina con diferente condición patológica. La clasificación articular y la edad se determinaron por inspección macroscópica. El grupo normal NN ( $n=47)$ no presentó daño visual de las estructuras articulares. Los grupos con daño articular fueron: a) un grupo con membrana sinovial congestiva sin daño del cartílago NA $(n=25), b)$ un grupo con daño del cartílago y con membrana sinovial normal $\mathrm{AN}(\mathrm{n}=23)$, y c) un grupo con daño del cartílago y con membrana sinovial congestiva AA $(n=15)$. El cartílago articular dañado presentó erosiones y múltiples líneas de roce y la membrana sinovial dañada presentó aspecto congestivo. No hubo diferencia significativa entre los grupos analizados. Sólo hubo tendencia a aumentar en el grupo NA $(44,4 \pm 32,6 \mu \mathrm{mol} / \mathrm{L})$, posiblemente asociado a la integridad del cartílago y a su mayor respuesta al estímulo proinflamatorio de la membrana sinovial congestiva. La concentración de nitrito tendió a disminuir en el grupo con daño de cartílago y membrana sinovial AA $(26,3 \pm 22,1 \mu \mathrm{mol} / \mathrm{L})$ que se explicaría por la pérdida de cartílago en este modelo in vivo.

\section{REFERENCIAS}

Adarmes H, F Vásquez, M Galleguillos, E González. 2008. Detección de glicosaminoglicanos de la matriz del cartílago articular en el líquido sinovial de carpo equino con fractura en esquirla. Arch Med Vet 40, 77-81.

Bird JLE, S May, MT Bayliss. 2000. Nitric oxide inhibits aggrecan degradation in explants cultures of equine articular cartilage. Equine Vet J 32, 133-139.

Clancy RM, AR Amin, SB Abramson. 1998. The role of nitric oxide in inflammation and immunity. Arthritis Rheum 2, 1141-1151.

Davis KL, E Martin, IV Turko, F Murad. 2001. Novel effects of nitric oxide. Annu Rev Pharmacol Toxicol 41, 203-236.

Ersoy Y, E Özerol, Ö Baysal, I Temel, RS MacWalter, Ü Meral, ZE Altay. 2002. Serum nitrite levels in patients with rheumatoid arthritis, ankylosing spondylitis, and osteoarthritis. Ann Rheum Dis 61, 76-78.

Förstermann U, J Boissel, H Kleinert. 1998. Expressional control of the constituve isoforms of nitric oxide synthase (NOS I and NOS II). Faseb J 12, 773-790.

Hashimoto S, RL Ochs, S Komiya, M Lotz. 1998. Linkage of chondrocyte apoptosis and cartilage degradation in human osteoarthritis. Arthritis Rheum 41, 1632-1638.

Häuselmann HJ, M Stefanovic-Racic, BA Michel, CH Evans. 1998. Differences in nitric oxide production by superficial and deep human articular chondrocytes: Implications for proteoglycan turnover in inflammatory joint disease. J Immunol 160, 1444-1448.

Infostat. 2004. Manual del usuario. Grupo Infostat, FCA, Universidad Nacional de Córdoba. Editorial Brujas, Córdoba, Argentina.

Jang D, G Murrell. 1997. Nitric oxide in arthritis. Free Radic Biol Med 24, 1511-1519.

Karan A, MA Karan, P Vural, N Erten, C Taşçıoğlu, C Aksoy, M Canbaz, A Öncel. 2003. Synovial fluid nitric oxide levels in patients with knee osteoarthritis. Clin Rheumatol 22, 397-399.

Kim DY, HW Taylor, RM Moore, DB Paulsen, D-Y Cho. 2003. Articular chondrocyte apoptosis in equine osteoarthritis. Vet $J 166,52-57$.

Lee MS, MCD Trindale, T Ikenoue, DJ Schurman, SB Goodman, RL Smith. 2002. Effects of shear stress on nitric oxide and matrix protein gene expression in human osteoarthritic chondrocytes in vitro. J Orthop Res 20, 556-561.

Lowry OH, NJ Rosebrough, AL Farr, RJ Randall. 1951. Protein measurement with the Folin's phenol reagent. J Biol Chem 193, 265-275.

Mazzetti I, B Grigolo, L Pulsatelli, P Dolzani, T Silvestri, L Roseti, R Meliconi, A Facchini. 2001. Differential roles of nitric oxide and oxygen radicals in chondrocytes affected by osteoarthritis and rheumatoid arthritis. Clin Sci 101, 593-599.

McIlwraith CW. 1996. General pathobiology of the joint and response to injure. In: McIlwraight CW, Trotter GW (eds). Joint disease in the horse. WB Saunders, Philadelphia, USA, Pp 40-70.

Miranda KM, MG Espey, DA Wink. 2001. A rapid, simple spectrophotometric method for simultaneous detection of nitrate and nitrite. Nitric Oxide $5,62-71$. 
Moshage H. 1997. Nitric oxide determinations: Much ado about NO - Thing? Clin Chem 43, 553-556.

Murrell GAC, D Jang, RJ Williams. 1995. Nitric oxide activates metalloproteinase enzymes in articular cartilage. Biochem Biophys Res Commun 206, 15-21.

Nagano T. 1999. Practical methods for detection of nitric oxide. Luminescence 14, 283-290.

Ralston SH. 1997. Nitric oxide and bone: What a gas! Br J Rheumatol 36, 831-838.

Spreng D, N Sigrist, A Schweighauser, A Busato, P Schawalder. 2001. Endogenous nitric oxide production in canine osteoarthritis: Detection in urine, serum and synovial fluid specimens. Vet Surg 30, 191-199.

Stichtenoth DO, JC Frölich. 1998. Nitric oxide and inflammatory joint disease. Br J Rheumatol 37, 146-257.

Tomita M, EF Sato, M Nishikawa, Y Yamano, M Inoue. 2001. Nitric oxide regulates mitochondrial respiration and fuctions of articular chondrocytes. Arthritis Rheum 44, 96-104.
Tung JT, J Venta, JO Caron. 2002. Inducible nitric oxide expression in equine articular chondrocytes: effects of antiinflammatory compounds. Osteoarthritis Cartilage 10, 5-12.

Van der Harst M, S Bull, PA Brama, AB Barneveld, PR van Weeren, $C$ van de Lest. 2006. Nitrite and nitrotyrosine concentrations in articular cartilage, subchondral bone, and trabecular bone of normal juvenile, normal adult, and osteoarthritic adult equine metacarpophalangeal joints. J Rheumatol 33, 1662-1667.

Van't Hof RJ, L Hocking, PK Wright, SH Ralston. 2000. Nitric oxide is a mediator of apoptosis in the rheumatoid joint. Rheumatology 39, 1004-1008.

Von Rechenberg B, CW McIlwraith, MK Akens, DD Frisbie, C Leutenegger, JA Auer. 2000. Spontaneous production of nitric oxide (NO), prostaglandin (PGE2) and neutral metalloproteinases (MMPs) in media of explant cultures of equine synovial membrane and articular cartilage from normal and osteoarthritic joints. Equine Vet $J 32,140-150$. 